\title{
AISLAMIENTO PULMONAR: EXCELENTE ALTERNATIVA ANTE LA FIBRILACIÓN AURICULAR
}

\author{
Alejandro Olaya Sánchez $M D^{*}$, José María Hennessey Sánchez MD**
}

\section{Resumen}

La fibrilación auricular (FA), es una arritmia caracterizada por la contracción rápida e irregular de las aurículas. La frecuencia varía entre 400 y 700 por minuto. Es la arritmia sostenida más frecuente en el hombre. Su prevalencia es alrededor de $0,5 \%$ según la población en estudio y alcanza hasta $8 \%$ en ancianos. Existe una considerable morbilidad, mortalidad y carga económica asociada con la FA, todo lo cual aumentará con la ampliación de la población de edad avanzada. Hasta hace poco el empleo farmacológico con bloqueo AV nodal utilizando agentes antiarrítmicos y anticoagulantes fue el pilar de la terapia. Aunque la cardioversión eléctrica se asocia con una alta tasa de éxito inmediato, la mayoría de los pacientes tienen recurrencias de FA, con sólo el 23\% restante en ritmo sinusal un año después del procedimiento. Ahora hay gran interés respecto al aislamiento de las venas pulmonares. Esta técnica consiste en la cartografía con catéteres multielectrodos de la región del ostium de las venas pulmonares, lo que permite identificar los segmentos de la unión venoatrial ocupados por las bandas musculares que se extienden desde la aurícula izquierda hasta las venas pulmonares. La aplicación de radiofrecuencia en dichas zonas permite la desconexión de las fibras musculares. Se realizó una revisión sobre la técnica apropiada a raíz del manejo de un paciente en el Hospital Cardiovascular del Niño de Cundinamarca.

Palabras clave: aislamiento pulmonar, fibrilación auricular, ablación con catéter de radiofrecuencia.

Abreviaturas: FA, fibrilación auricular; EVC, evento vascular cerebral; VP, venas pulmonares.

\section{PULMONARY ISOLATION: AN EXCELENT ALTERNATIVE TO ADDRESS ATRIAL FIBRILLATION}

\section{Abstract}

Atrial fibrillation (AF) is a fast and irregular heart arrhythmia that starts in the atria. The rate may range from 400 to 700 beats per minute. It is the most common continuous arrhythmia in humans. Its prevalence is around $0.5 \%$ according to the study population and reaches up to $8 \%$ in elders. AF-related morbidity, mortality and economic burden are significant and will increase as elderly population increases. Until not long ago, therapy was based on pharmacological AV node blockage using antiarrhythmic and anticoagulant agents. Although electrical cardioversion is associated with a high immediate success rate, the majority of patients may have relapses with only the remaining $23 \%$ maintaining a normal sinus rhythm a year after the procedure. Recently there has been great interest in pulmonary vein isolation. This technique consists on inserting a multielectrode catheter and mapping the os of the pulmonary veins to identify the venoatrial attachment occupied by muscle bands extending from the left atrium to the pulmonary veins. Disconnection of these muscle fibers is enabled by applying radiofrequency energy to these areas. A review of the appropriate technique was conducted when handling one patient at the Hospital Cardiovascular del Niño de Cundinamarca.

Key words: pulmonary isolation, atrial fibrillation, radiofrequency catheter ablation.

Fecha recibido: mayo 23 de 2011 - Fecha aceptado: agosto II de 2011

* Jefe del Departamento de Electrofisiologia del Hospital Cardiovascular del Niño, Cundinamarca, y del Hospital de San José, Bogotá DC. Colombia.
** Residente II de Cirugía Cardiovascular, Hospital de San José, Fundación Universitaria de Ciencias de la Salud, Bogotá DC. Colombia. 


\section{Introducción}

La FA es una arritmia caracterizada por la contracción rápida e irregular de las aurículas. La frecuencia de contracción varía entre 400 y 700 por minuto. Es la arritmia sostenida más frecuente en el hombre. Su prevalencia es alrededor de $0,5 \%$ según la población en estudio y alcanza hasta $8 \%$ en los ancianos. El estudio Framingham demostró una prevalencia de FA del 12\% en los mayores de 70 años en comparación con el 5\% entre 60 y $70 .{ }^{1,2}$ La consecuencia más devastadora es el evento vascular cerebral (EVC) como resultado de tromboembolismo proveniente de la aurícula izquierda, cuya frecuencia alcanza 5\% por año. Uno de cada seis EVC isquémicos ocurre en pacientes con FA, incluyendo ataques transitorios y EVC silente detectado mediante radiografía. ${ }^{3,4}$

Durante un siglo se ha sabido que la FA coexiste en presencia de varias formas de enfermedad cardíaca. En individuos con FA aislada se han encontrado ciertas arritmias precursoras, en especial flutter auricular, taquicardias auriculares focales y taquicardia supraventricular de reentrada como la taquicardia nodal de reentrada y la ortodrómica recíproca. Se sabe también que la remodelación auricular eléctrica y mecánica, como consecuencia de la arritmia, tiende a perpetuar la FA. Este proceso es el resultado del incremento de la frecuencia auricular; sin embargo, la isquemia auricular, factores neurohumorales y autonómicos pueden contribuir de igual manera. ${ }^{5}$ Los mecanismos básicos electrofisiológicos de la FA son la reentrada y las descargas rápidas de focos auriculares ectópicos. ${ }^{6}$ La hipótesis de la reentrada múltiple propone que el fraccionamiento de los frentes de ondas reentrantes genera ondas reentrantes "hijas" que se autoperpetúan. Este circuito de reentrada es errante y es constante el cambio de localización de un foco ectópico de descarga muy rápida, por dos focos ectópicos que laten a frecuencias distintas; el número de ondas depende de la duración del periodo refractario, de la masa del tejido y de la velocidad de conducción en los diferentes sectores de las aurículas.

Desde el punto de vista terapéutico existen varias alternativas para la conversión de FA a ritmo sinusal y su mantenimiento. La amiodarona se ha incorporado de manera definitiva dentro de las posibilidades para la reversión de la FA. Otras opciones terapéuticas incluyen betabloqueadores, bloqueadores de los canales de calcio, anticoagulación y en ocasiones cardioversión. ${ }^{6}$ Sin embargo, cerca del 50\% de los pacientes tienden a recurrir al año de haber iniciado la terapia farmacológica. El tratamiento de la FA sintomática en su forma crónica incluye la ablación percutánea transcatéter, con probabilidades de éxito alrededor del 70\%, para el porcentaje restante existen alternativas quirúrgicas que incluyen la ablación epicárdica con microondas y la cirugía de Maze. ${ }^{1,7.8}$.

Haissaguerre estableció que el papel de las VP en el mecanismo arritmogénico se da por la presencia de actividad eléctrica, la cual se asocia con la génesis de latidos ectópicos y el inicio de paroxismos frecuentes de FA. ${ }^{6}$ Se ha explicado la génesis de esta actividad por la capacidad del músculo alrededor de la porción proximal de las VP de generar potenciales de acción secundarios a corrientes iónicas activadas por estiramiento al paso del flujo sanguíneo. ${ }^{9}$ Las fibras musculares estriadas son abundantes en las VP superiores, además se observan conexiones eléctricas localizadas, de predominio en los segmentos inferiores de las VP superiores y en los segmentos superiores de las inferiores. ${ }^{10,11}$ Por otro lado, la pared muscular de la aurícula izquierda puede extenderse hacia arriba unos pocos centímetros alrededor de las VP, lo que puede crear diferencias significativas en el diámetro, grosor de la pared y extensión de tejido cardíaco alrededor de las VP. ${ }^{6,12,13}$ El estudio realizado por Hamabe y colaboradores en VP de perros, reveló que la orientación compleja de fibras musculares provee bases anatómicas para producir alteraciones en la conducción a nivel de su unión en la aurícula izquierda. ${ }^{14}$

La presencia de latidos ectópicos en las VP incrementa la posibilidad de que estas contengan células marcapaso. ${ }^{15}$ Los estudios en tejidos de embriones humanos encuentran que presentan el mismo origen de otras células cardíacas de conducción y sirven como foco de automatismo. ${ }^{12,14}$ Cheung demostró por primera vez en cerdos, que las VP poseen actividad eléctrica espontánea. La supresión con procainamida 
de su actividad focal en modelos caninos, sugiere que la automaticidad tiene un rol en la actividad arritmogénica de las VP..$^{12,14,16} \mathrm{El}$ aislamiento de grupos miocárdicos debido a fibrosis, reduce el acoplamiento entre los miocitos y disminuye la inhibición eléctrica, incrementando el automatismo. ${ }^{12,14,16}$ Se han observado largos períodos refractarios efectivos en las VP proximales más que en las distales, lo que sugiere que al bloquear los impulsos originados desde estas últimas, se puede reducir la posibilidad de desarrollar FA.

Hassink y colaboradores realizaron estudios sobre histología en pacientes con y sin FA y concluyó que las VP se caracterizaban por discontinuidad en la conducción, hipertrofia y fibrosis, lo que sugería un sustrato arritmogénico. ${ }^{6,13,14}$ Como propiedades electrofisiológicas del músculo en las VP se ha descrito la presencia de conducción disminuida y períodos refractarios cortos en algunas VP arritmogénicas, lo que sugiere un mecanismo de reentrada en la génesis de esta actividad eléctrica ectópica. Se han encontrado estos circuitos de reentrada que pudieron haber sido causados por conducción anisotrópica y heterogeneidad en la repolarización. ${ }^{17}$

En algunos pacientes la FA parece tener origen focal, que estaría ligado de manera predominante a las VP u originarse en otros sitios, tales como venas cavas superiores, ligamento de Marshall, seno coronario o en el mismo tejido auricular. ${ }^{18} \mathrm{La}$ importancia de este origen radica en la posibilidad de tratamiento curativo mediante la ablación por catéter. El objetivo de este procedimiento en las VP consiste en eliminar la posibilidad de que exista actividad eléctrica (ablación focal), o evitar que dicha actividad se pueda propagar hacia la aurícula izquierda (aislamiento de venas pulmonares). ${ }^{19,20}$ La primera técnica sobre focos ectópicos localizados en la desembocadura de las VP en pacientes con FA paroxística, ha despertado el interés por los métodos de imagen capaces de ofrecer una adecuada información anatómica de las VP. ${ }^{21}$ Se sabe que la ablación se realiza a través de un mapeo perimétrico de las VP, de tal forma que el conocimiento de la anatomía de las VP, en especial el ostium, es de gran importancia para la adecuada selección del catéter de mapeo y determinar que todos los orificios de las VP sean evaluados.

La venografía pulmonar se suele realizar con frecuencia previa a la ablación, con el fin de determinar el tamaño, la forma y la posición del ostium venoso. Sin embargo, presenta algunas limitaciones como la magnificación del ostium, así como una pobre visualización de cada VP en el plano transverso. Una vez determinada la anatomía de las VP se procede a la ablación, la cual se realiza a través de un abordaje transeptal para alcanzar la aurícula izquierda, sea a través del foramen oval permeable o de una punción transeptal. Se identifican las desembocaduras de las VP mediante angiografía o ultrasonido intracardíaco, este último examen con ventaja sobre la fluoroscopia, ya que provee imágenes de alta resolución y una visualización directa del endocardio, lo que facilita la colocación de catéteres y proporciona información detallada sobre las variaciones anatómicas de las VP.

El uso del ultrasonido intracardíaco sirve además para valorar la estenosis de las VP, que es una complicación posterior al procedimiento. El uso de mapeo endocárdico por impedancia ha logrado disminuir este riesgo después de la ablación circunferencial del ostium, ya que ayuda a distinguir el tejido de la aurícula izquierda y del ostium de la VP. Se identifica la presencia de actividad eléctrica anormal en cada una de las VP, mediante cartografía guiada por fluoroscopia al introducir un catéter decapolar en el ostium de las VP, o con electroanatómica no fluoroscópica (sistemas Carto, LocaLisa). ${ }^{22}$ Se han desarrollado técnicas para la desconexión de las VP mediante cartografía circunferencial, longitudinal y electroanatómica. La radiofrecuencia está indicada cuando hay acción refractaria a los fármacos, recurrencia de los paroxismos, paroxismos sintomáticos o afección progresiva en la calidad de vida y ante el compromiso de la estructura atrial.

Se ha descrito que la ablación circunferencial tiene como ventaja el menor riesgo y la ausencia de estenosis de las VP. De igual manera, se ha asociado con mayor tasa de éxito cuando se usa el catéter irrigado. La presencia de dobles electrocardiogramas localizados en las VP demuestra la presencia de actividad eléctrica 
anormal dentro de la VP, denominada "potencial de la vena pulmonar", sugiriendo que se trata de una vena profibrilatoria. La tasa de éxito aumenta cuando al finalizar haya menos VP con actividad eléctrica.

\section{Caso}

Paciente masculino de 48 años de edad, con antecedente de palpitaciones rápidas, irregulares y casi permanentes desde hace cerca de siete años. En 1998, en otro centro se le realizó un estudio electrofisiológico descartándose la presencia de taquicardia nodal o vías anómalas. El diagnóstico de ese momento fue de taquicardia auricular multifocal con episodios de FA. Con estos antecedentes fue remitido para su diagnóstico y tratamiento.

El electrocardiograma revelaba una taquicardia auricular con episodios de FA. Los estudios complementarios (radiografía de tórax, laboratorio completo, función tiroidea y ecocardiograma bidimensional con Doppler) no pusieron de manifiesto alteraciones cardíacas ni extracardíacas. La prueba de esfuerzo de febrero de 2010 no mostró alteración electrocardiográfica para insuficiencia coronaria (Figura 1).

La prueba de Holter de 24 horas previa al aislamiento pulmonar de junio de 2010 evidenció fibrilación auricular con episodios intermitentes de ritmo sinusal y frecuencia cardíaca promedio de $73 \mathrm{x}$ minuto (Figura 2). Fue medicado en forma alterna con fármacos antiarrítmicos (amiodarona), sin control efectivo.

\section{Medicada en forma}

Ante la falla en el control de la fibrilación auricular (a pesar de llevar más de tres meses de tratamiento con antiarrítmico del grupo III), se decide llevar a aislamiento de VP. Se solicita TAC de corazón multicorte gatillado para integración (fusión) con el sistema de mapeo electroanatómico Navx Ensite (St. Jude Medical, endocardial solutions).

El TAC de tórax con contraste muestra tres VP derechas: principal superior, accesoria adyacente a la inferior y la inferior principal (Figura 3). Se inicia procedimiento por medio de punción yugular derecha, se avanza un catéter tetrapolar defectable a la aurícula derecha que se aloja en el tercio distal del seno coronario, fijándolo como referencia. Por doble vía venosa femoral derecha, bajo efecto de anestesia local, con aguja de punción transeptal y mediante técnica de Seldinger se coloca introductor Mullins $8.0 \mathrm{~F}$ y un introductor de $S R O$ de $S t$ Jude de 8.5 F. Previo al estudio el paciente presentaba extrasístoles auriculares y episodios de FA que se resolvían en forma espontánea (Figura 4). Durante el procedimiento el paciente desarrolló FA que se sostuvo (Figura 5).

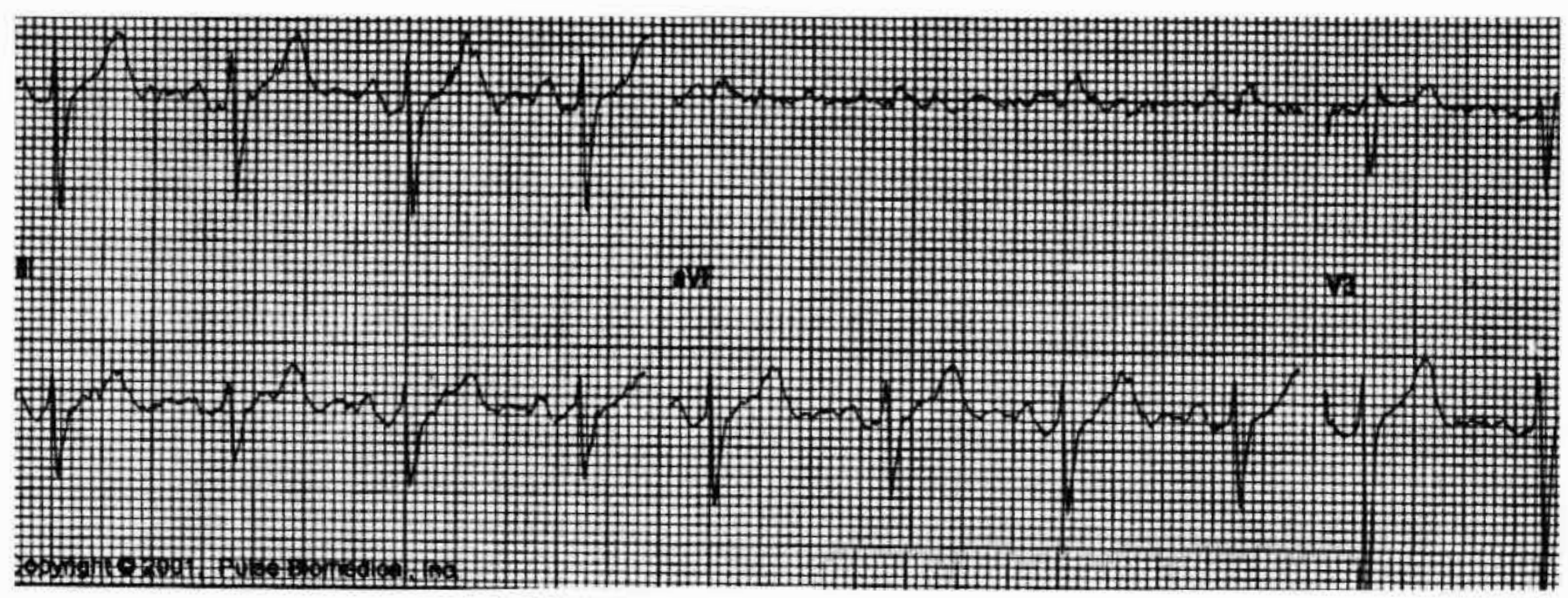

Figura I. Prueba de esfuerzo máxima al 90\% clase funcional IA II METS. Negativa para insuficiencia coronaria. 


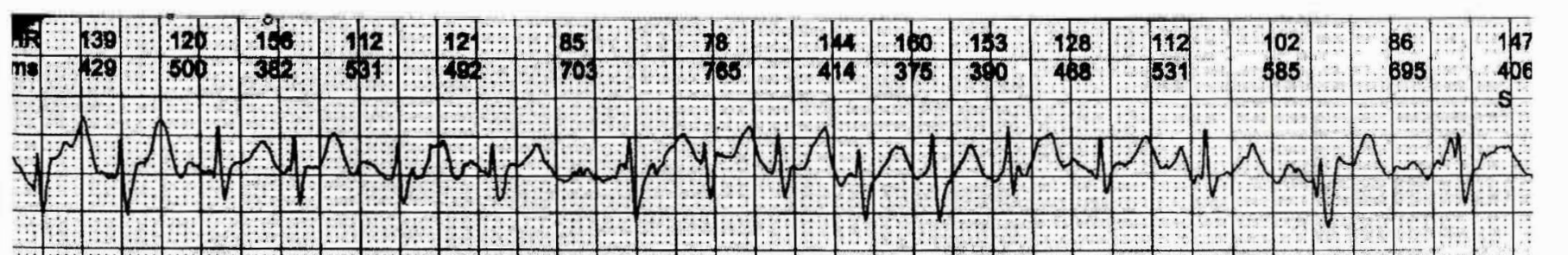

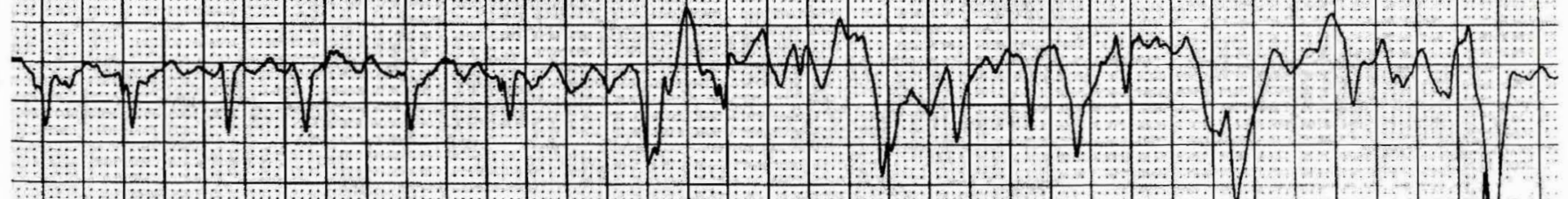

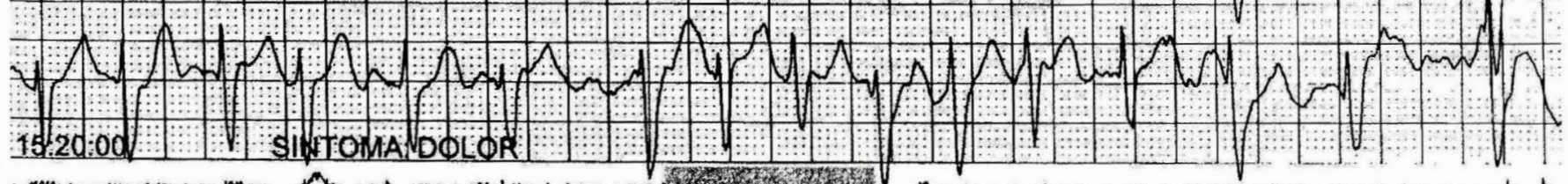

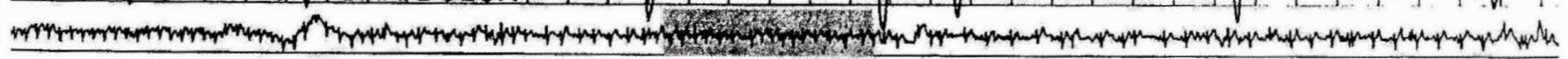

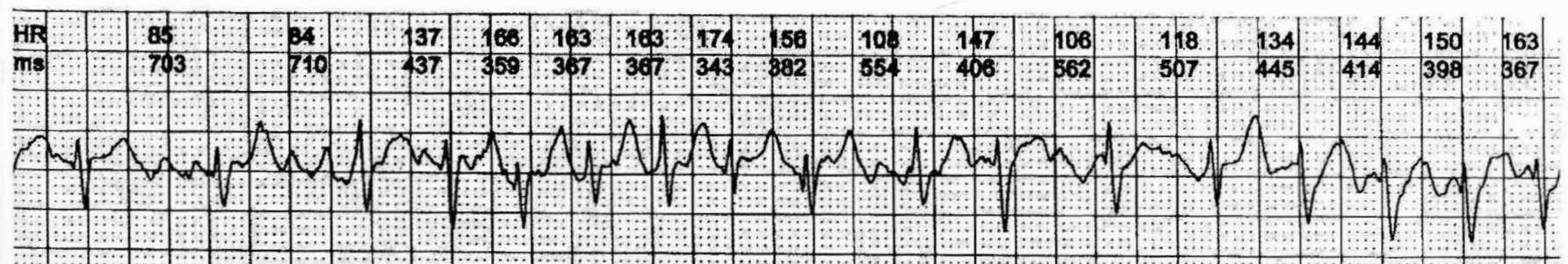

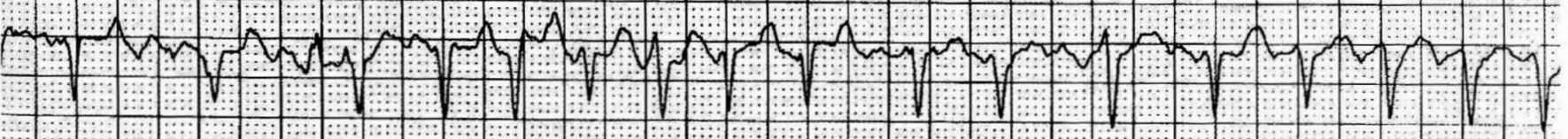

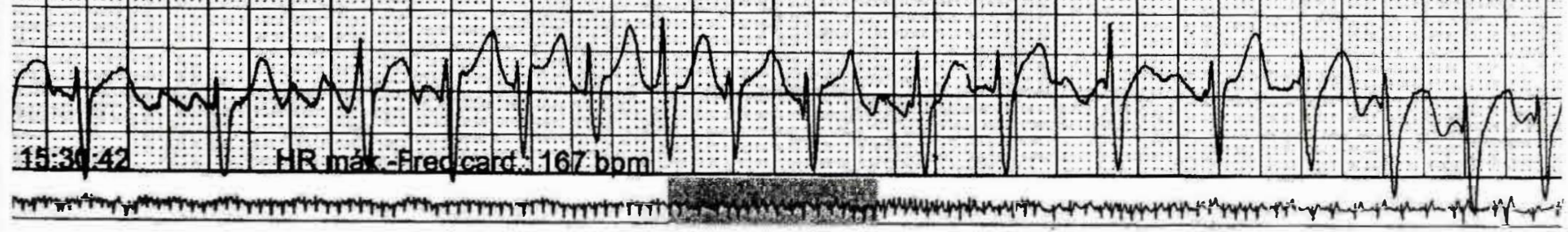
Tat

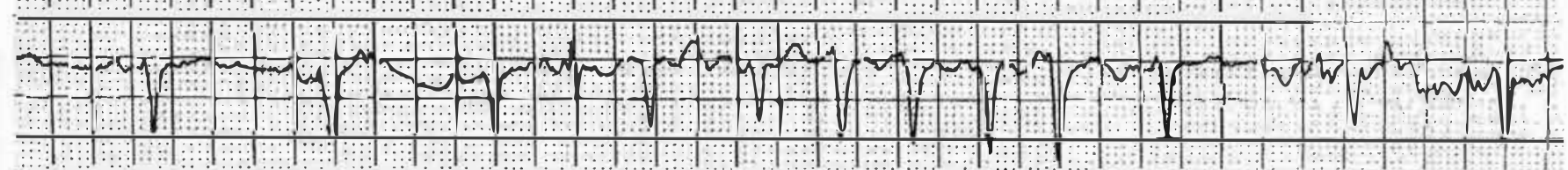

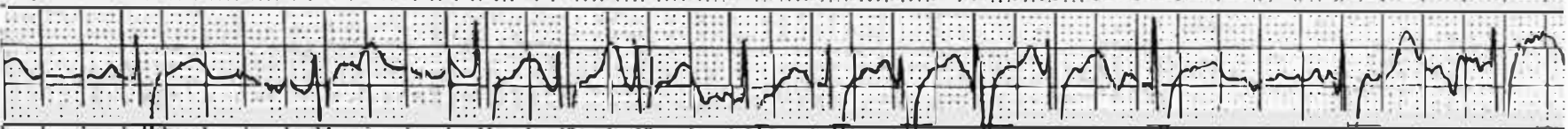

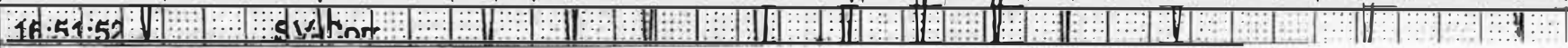

Figura 2. Prueba de Holter de junio de 2010 con ritmo de fibrilación auricular. 

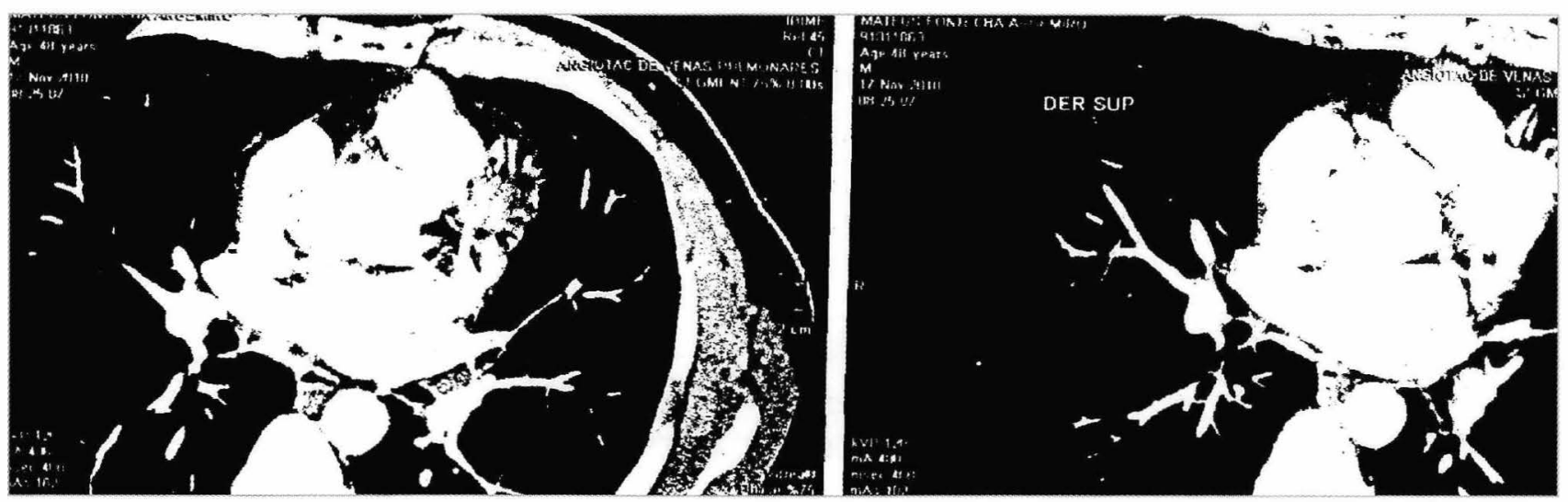

Figura 3.TAC de tórax con contraste.

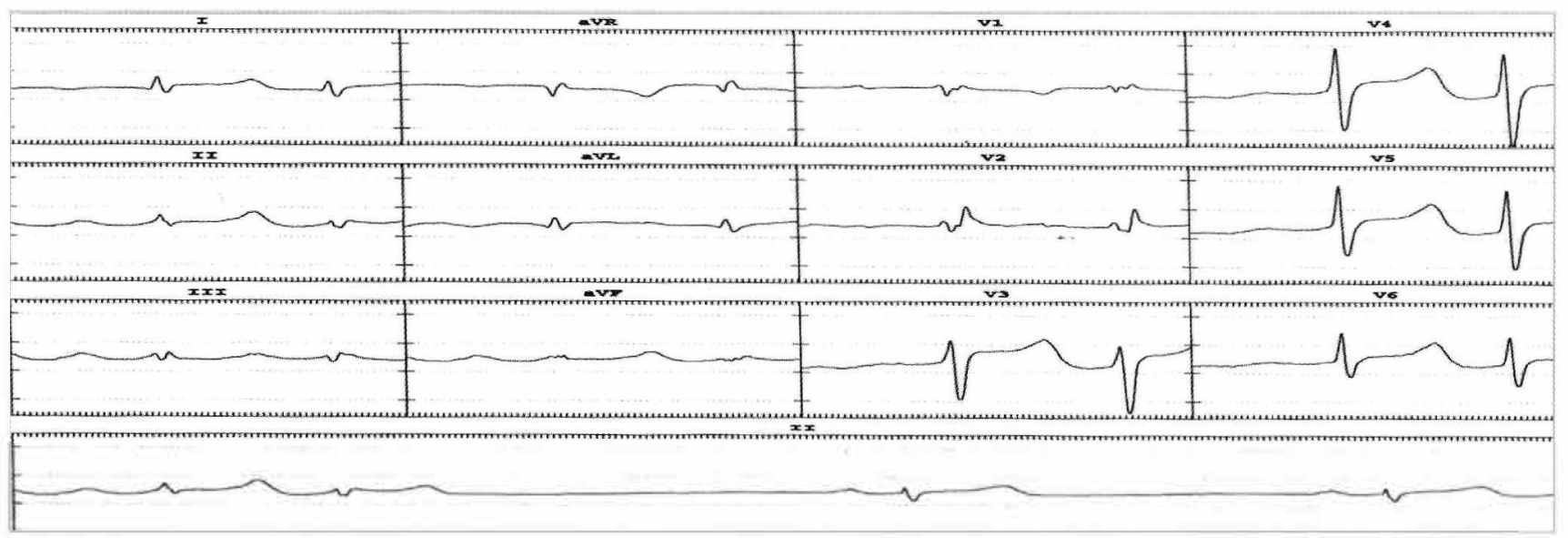

Figura 4.Trazado electrocardiográfico previo al inicio del procedimiento.

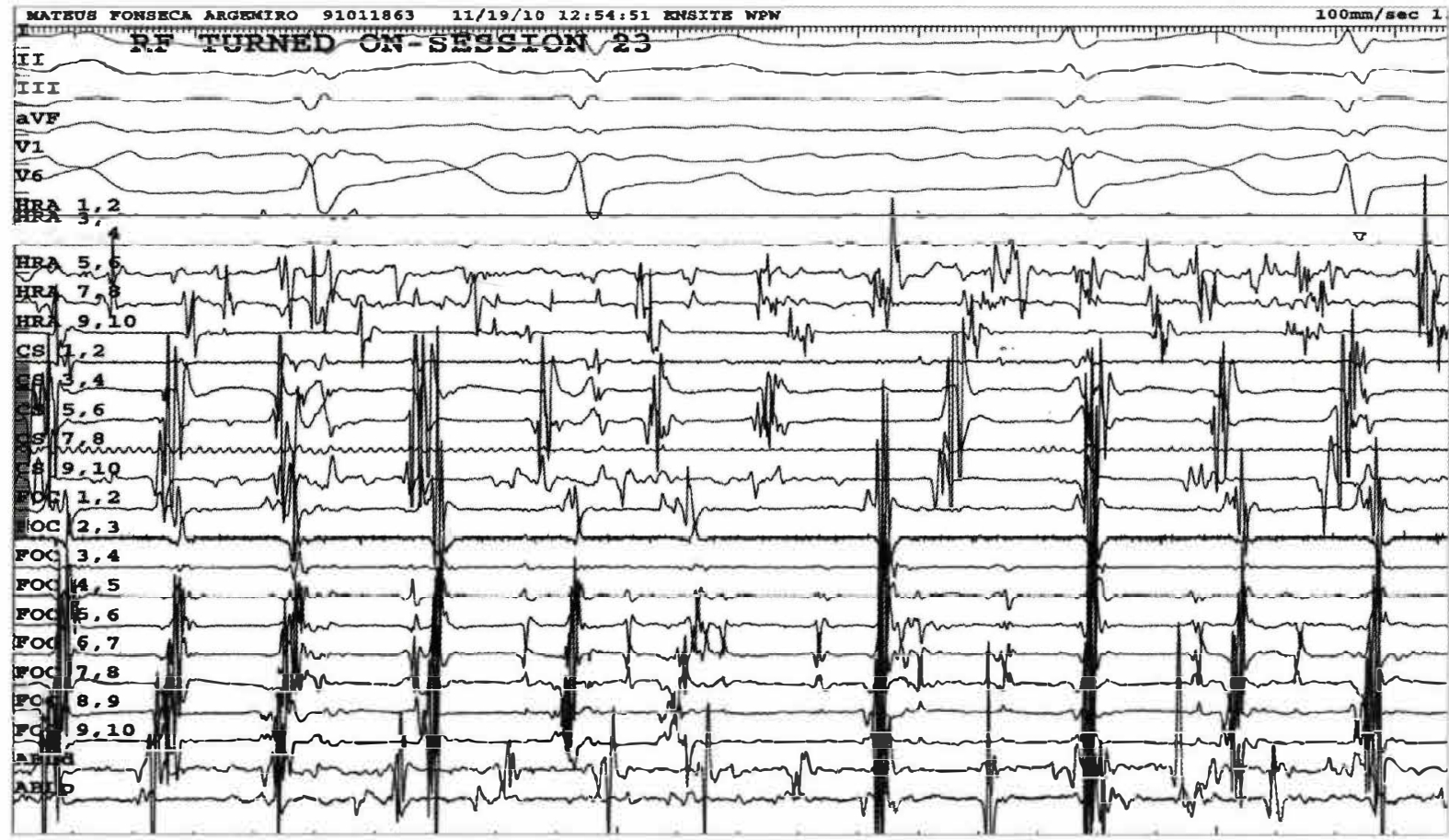

Figura 5. Obsérvese en catéter circular (Foc I hasta 10) actividad auricular rápida desordenada. 
Se realiza mapeo electroanatómico de la aurícula izquierda e integración del mismo a las imágenes de TAC, con aplicación continua de radiofrecuencia antral alrededor de las cuatro VP. Al completar aislamiento de las venas izquierdas se observa interrupción de la fibrilación auricular (Figuras 6, 7, 8 y 9).

El control de la prueba de Holter veinte días después del aislamiento pulmonar mostró extrasistolia supraventricular con episodios autolimitados de taquicardia auricular, sin FA (Figura 10). El paciente en el control refiere notoria mejoría con pocas palpitaciones y mejoría en su calidad de vida.

\section{Conclusión}

La FA es la arritmia más común y demandante, tiene riesgo elevado de morbimortalidad, de tal forma que el objetivo del tratamiento está encaminado a restaurar el ritmo sinusal para sobrevivir y tener mejor calidad

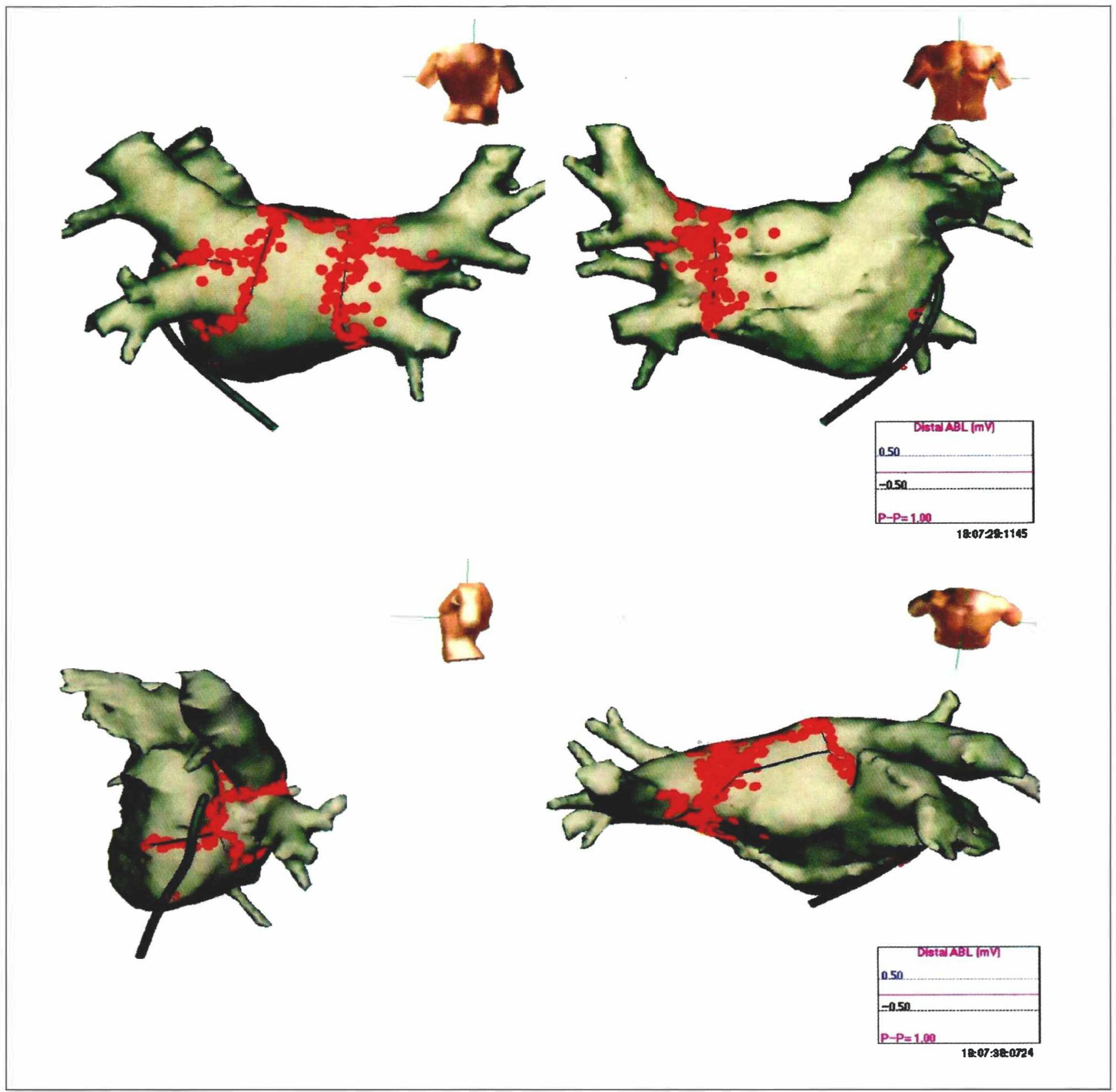

Figura 6. Mapeo alrededor de las cuatro VP. 


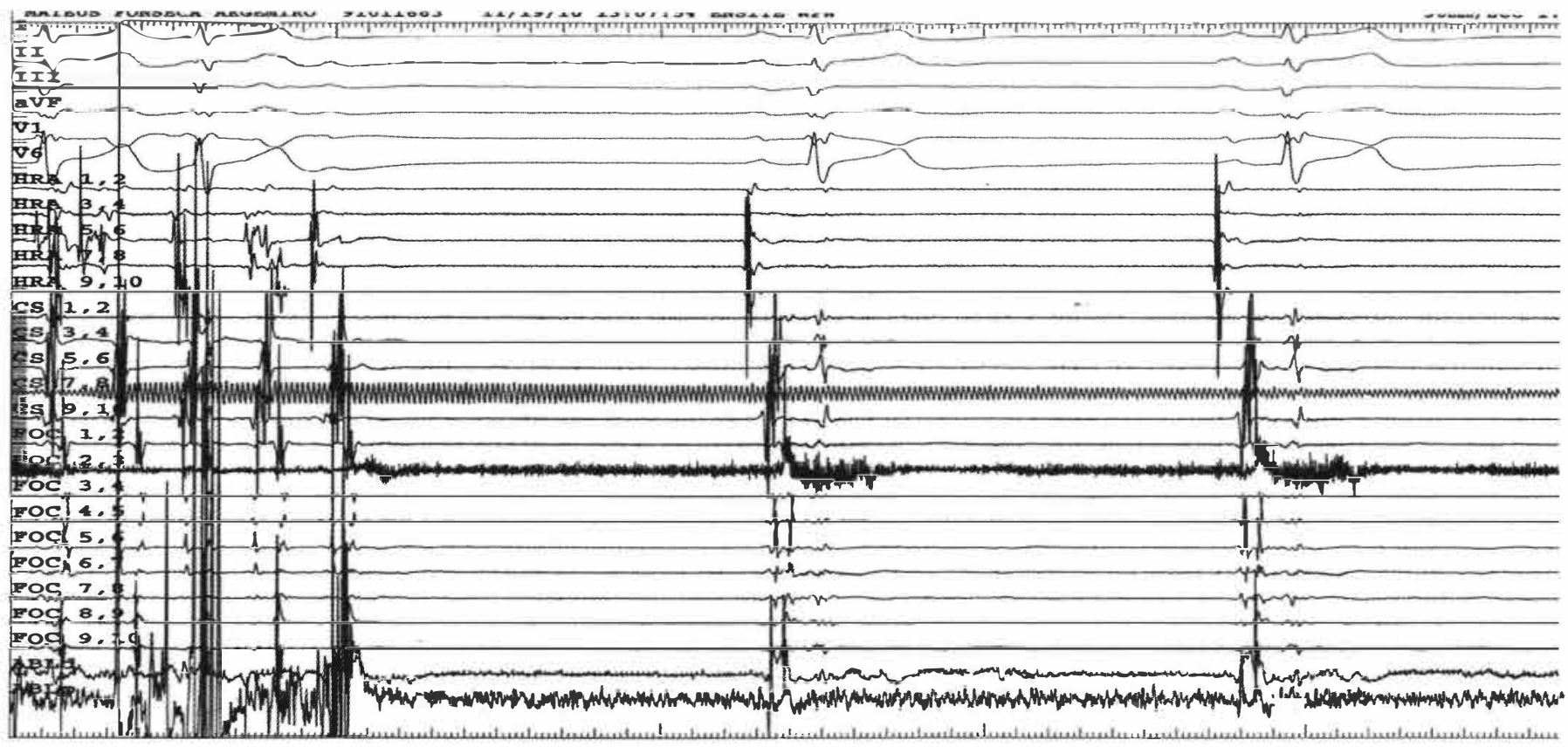

Figura 7. Obsérvese un silencio eléctrico dentro de los antros de las VP izquierdas (Foc I al 10), mostrando que no hay conducción dentro de los círculos de ablación.

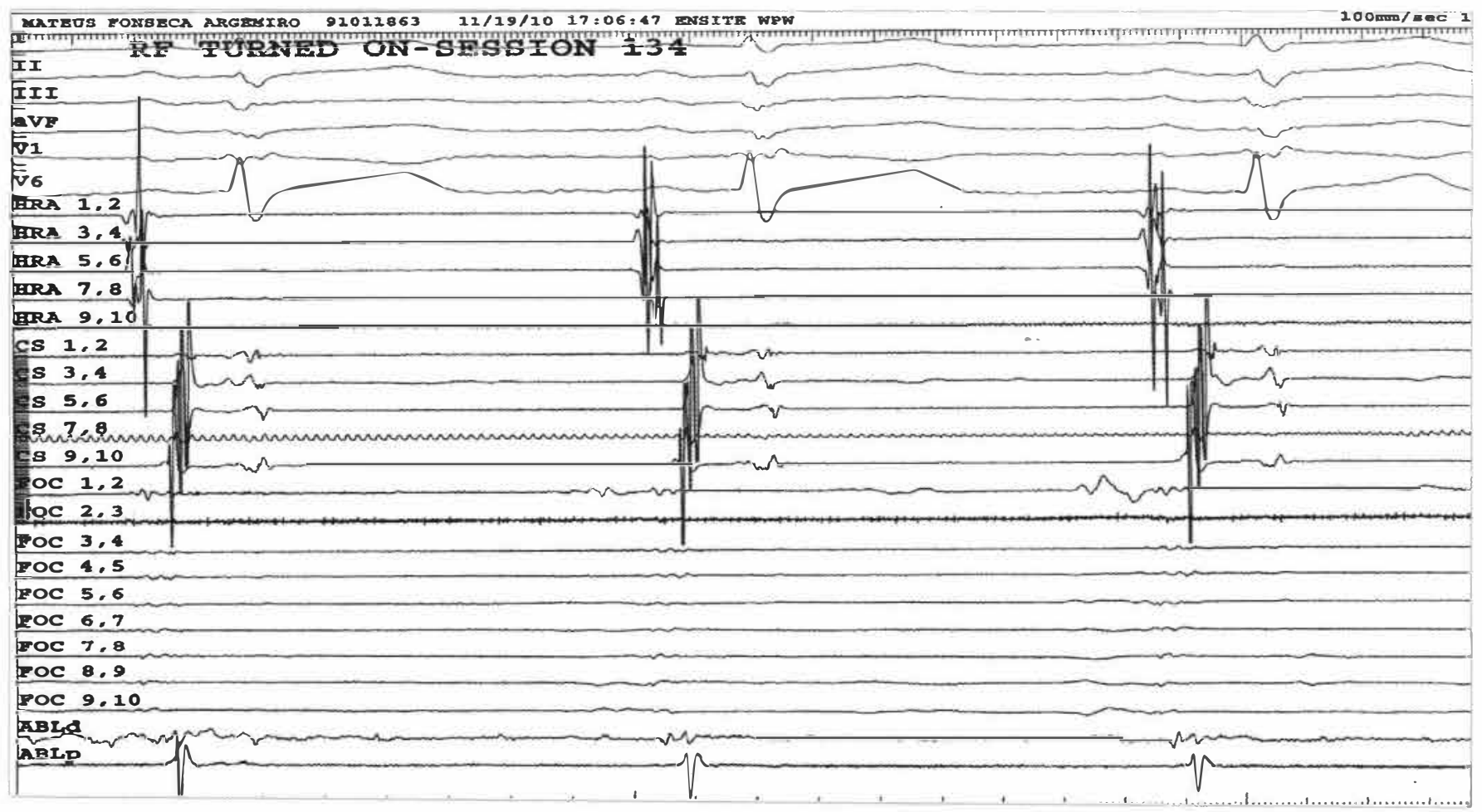

Figura 8. Se estimula por fuera de los círculos de ablación observando que no hay conducción dentro de las VP, demostrando el aislamiento dentro de las VP. 


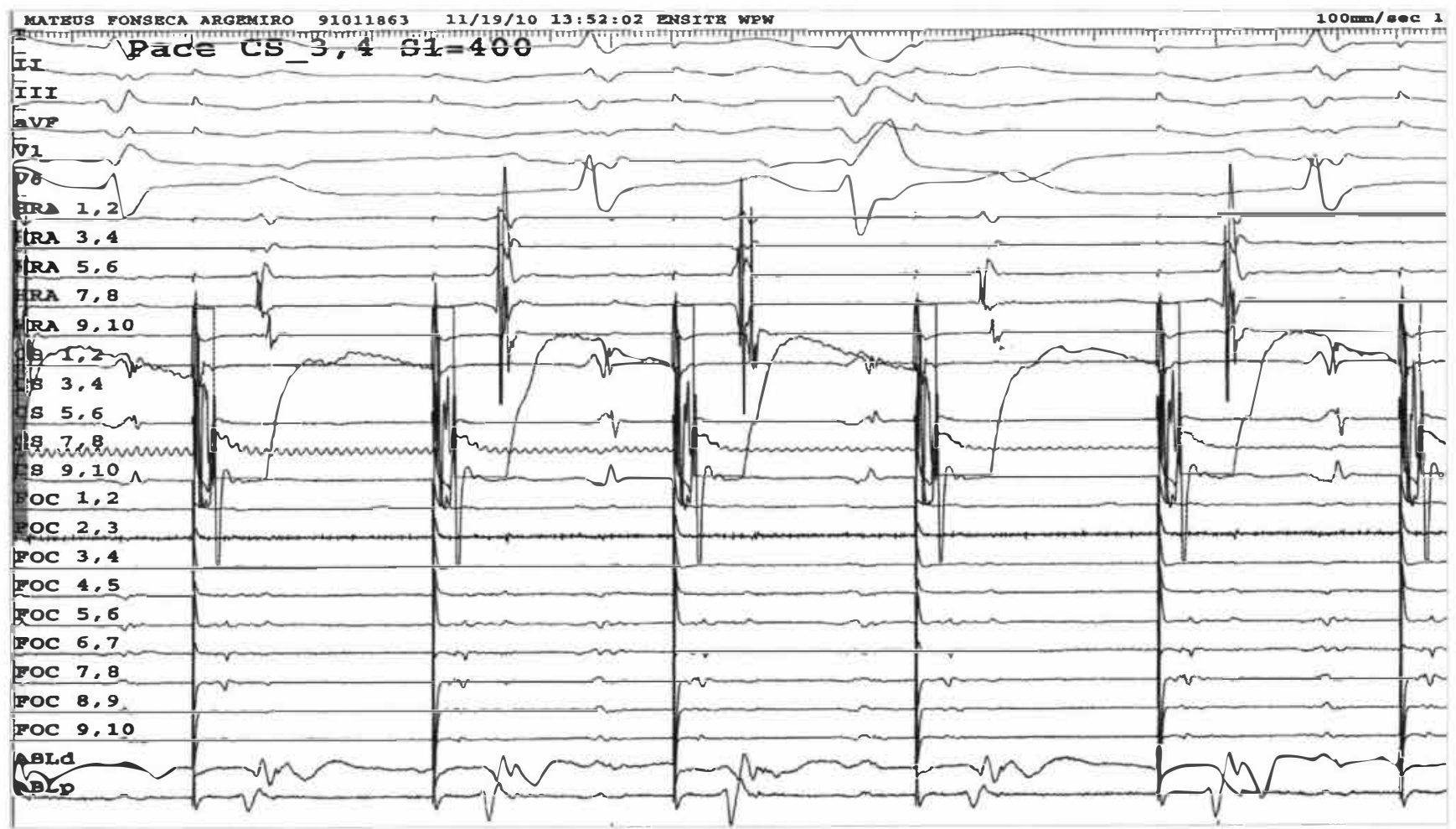

Figura 9. Se completan dos círculos antrales de ablación alrededor de las cuatro VP con aplicación sobre la carina, hasta observar el aislamiento de todas. Se termina el procedimiento luego de ocho horas de duración, con el aislamiento exitoso de las VP, sin inducción de arritmia POP.

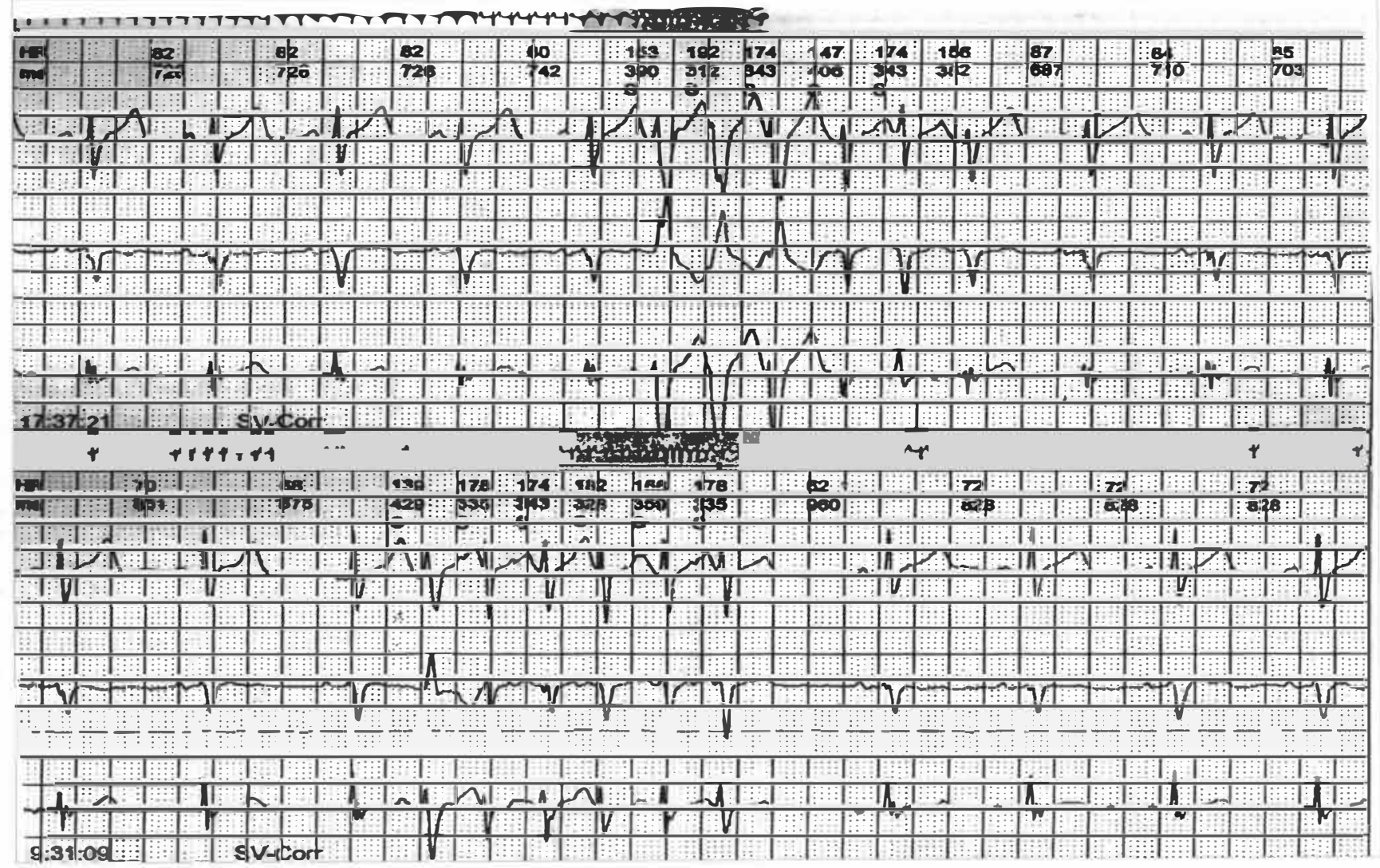

Figura 10. Prueba de Holter realizada al paciente veinte días después del procedimiento sin evidencia de FA. 
de vida. Con el tratamiento farmacológico la arritmia tiende a recurrir en un año hasta en $50 \%$ de los pacientes. Por lo anterior, se ha originado la búsqueda de alternativas terapéuticas, no farmacológicas, que ayuden a retornar al ritmo sinusal.

El tratamiento para la forma crónica de la fibrilación incluye terapia de ablación percutánea transcatéter con probabilidades de éxito cercanas al 70\%, la ablación quirúrgica epicárdica y en ocasiones el procedimiento de Maze, cada una de estas con probabilidades de éxito similar alrededor del $70 \%$. El hecho de que exista dominancia de la aurícula izquierda sobre la región de las VP en el disparo o en el mantenimiento de la FA, ha motivado el desarrollo de nuevas técnicas de ablación por radiofrecuencia. De tal forma que la cura de la FA es posible ahora mediante avances terapéuticos con radiofrecuencia a través de catéter, en los pacientes en que se demuestre actividad eléctrica anormal en las VP. Los últimos años han marcado el comienzo de una nueva era en la terapia de la FA. Para los pacientes con FA paroxística, segmentaria y circunferencial, parecen ser comparables a largo plazo las tasas de éxito y las bajas complicaciones. Sin embargo, cuando hay FA persistente o permanente, la ablación circunferencial utilizando $3 D$ electroanatomic cartografía parece tener mayor éxito. Los pacientes con FA que son candidatos son aquellos que presentan FA sintomática, a pesar de esfuerzos mínimos y aquellos que cursaron cardiopatía estructural. El futuro de la terapia de ablación de FA podría eliminar el disparador de la FA y alterar el sustrato que permite el mantenimiento de la arritmia. Nuevos diseños de catéteres y fuentes de energía alternativa se encuentran en investigación para mejorar la seguridad, eficiencia y tasa de éxito de la ablación por catéter. Este es un procedimiento que va alcanzando su madurez, como lo demuestra la inclusión en las guías de práctica clínica publicadas en 2006 por las sociedades Europea y Americana de Cardiología, como alternativa de la terapia farmacológica.

\section{Referencias}

1. Elizari MV. Consenso de fibrilación auricular. Rev. argent. de cardiol. 2005. 73(6): 469-85

2. Kannel WB, Abbott RD, Savage DD, McNamara PM. Coronary heart disease and atrial fibrillation: the Framingham Study. Am Heart J. 1983;106:389-96.
3. Kato R, Lickfett L, Meininger G, Dickfeld T, Wu R, Juang G, et al. Pulmo nary vein anatomy in patients undergoing catheter ablation of atrial fibrillation lessons learned by use of magnetic resonance imaging. Circulation. 2003;107 2004-10.

4. Oral H, Pappone C, Chugh A, Good E, Bogun F, Pelosi F, et al. Circumferential Pulmonary - vein ablation for chronic atrial fibrillation. N Engl J Med. 2006;354:934-41.

5. Wyndham CR. Atrial fibrillation: The most common arrhythmia. Texas Heart Institute Joumal. 2000;27:257-67.

6. Haissaguerre M, Jais P, Shah DC, Takahashi A, Hocini M, Quiniou G, et al. Spontaneous initiation of atrial fibrillation by ectopic beats originated in the pulmonary veins. NEJM. 1998;339:659-66.

7. Márquez MF, C•lín L, Guevara-Valdivia ME, Iturralde P, Castañeda R, Villavicencio R, et al. Ablación segmentaria del ostium de la vena pulmonar superior izquierda para eliminar actividad eléctrica anormal generada de fibrilación atrial paroxística idiopática. Arch Cardiol Mex. 2003;73:124-8.

8. Fuster V, Ryden LE, Asinger RW, Cannom DS, Crijns HJ, Frye RL, et al. ACC/ AHA/ESC guidelines for the management of patients with atrial fibrillation. A report of the American College of Cardiology/American Heart Association Task Force on Practice Guidelines and the European Society of Cardiology Committee for Practice Guidelines and Policy Conferences (Committee to develop guidelines for the management of patients with atrial fibrillation) developed in collaboration with the North American Society of Pacing and Electrophysiology. Eur Heart J. 2001;22:1852-923.

9. Vargas J. Tratado de Cardiología. Sociedad Mexicana de Cardiología. México. 2006;320-7.

10. Calkins H, Yong P, Miller JM, Olshansky B, Carlson M, Saul JP, et al. Catheter ablation of accessory pathways, atrioventricular nodal reentrant tachycardia, and the atrioventricular junction: final results of a prospective, multicenter clinical trial. The Atakr Multicenter Investigators Group. Circulation. 1999;99(2):262-70

11. Wijffels MCEF. Kirchhof CJHJ, Doland R, Allessie MA. Atrial fibrillation begets atrial fibrillation. A study in awake chronically instrumented gotas. Circulation. 1995;92:1954-68.

12. Chen YJ, Chen SA. Electrophysiology of pulmonary veins. J Cardiovasc Electrophysiol. 2006;17:220-4.

13. Wellens HJ. Pulmonary vein ablation in atrial fibrillation: hype or hope? Circulation. 2000;102:2562-4

14. Chen SA, Hsieh MH, Tai CT, Tsai CF, Prakash VS, Yu WC et al. Initiation of atrial fibrillation by ectopic beats originating from the pulmonary veins: electrophysiological characteristics, pharmacological responses, and effects of radiofrequency ablation. Circulation. 1999;100:1879-86

15. Silva R, Mont LI, Berruezo A, Fosch X, Wayar L, Alvarenga N, et al. Ablación por radiofrecuencia para el tratamiento de la fibrilación auricular focal a través de cartografía circunferencial y aislamiento segmentario de las venas pulmonares. Rev Esp Cardiol. 2003;56(4):361-7.

16. Stabile G, Turco P, La Rocca V, Nocerino P, Stabile E. De Simone A. Is pulmonary vein isolation necessary for curing atrial fibrillation? Circulation. 2003; 108:657-60

17. Hamabe S, Okuyama Y, Miyauchi Y, Zhou S, Pak HN, Karagueuzian HS, et al. Correlation between anatomy and electrical activation in canine pulmonary veins. Circulation. 2003;107:1550-5.

18. Ouyang F, Bansch D, Ernst S, Schaumann A, Hachiya H, Chen M. Complete isolation of left atrium surrounding the pulmonary veins: new insights from the double-Lasso technique in paroxysmal atrial fibrillation. Circulation. 2004;110:2090-6.

19. Chung MK, Martin DO, Sprecher D, Wazni O, Kanderian A, Carnes CA, et al. C-reactive protein elevation in patients with atrial arrhythmias: Inflammatory mechanisms and persistence of atrial fibrillation. Circulation. 2001;104:2886-91.

20. Velarde JL, Martellotto R, Scanavaca M, Arévalo A, Colque R, Jimenez M, et al. Ablación de las venas pulmonares en la fibrilación auricular. Experiencia inicial. Rev Esp Cardiol. 2002;55(5):541-5.

21. Haissaguerre M, Shah DC, Jais P, Hocini M, Yamane T, Deisenhofer I. Mapping - guided ablation of pulmonary veins to cure atrial fibrillation. 2000 Nov 2;86(9A):9K-19K 2000 Nov 2; 86(9A):9K-19K

22. Pappone C, Rosanio S, Oreto G, Tocci M, Gugliotta F, Vicedomini G, et al. Circumferential radiofrequency ablation of pulmonary vein ostia: A new anatomic approach for curing atrial fibrillation. Circulation. 2000:102:2619-28. 